\title{
Electron Transit Time Measurements of 5-inch Photomultiplier Tubes
}

\author{
T. Richards, J. Peatross, M. Ware, L. Rees \\ Dept. of Physics and Astronomy, Brigham Young University, Provo, Utah 84602, USA
}

5 Abstract

We investigated the uniformity of electron transit times for two 5-inch photomultiplier tubes: the Hamamatsu R1250 and the Adit B133D01S. We focused a highly attenuated short-pulse laser on the tubes while they were mounted on a programmable stage. The stage translated the tubes relative to the incident beam so that measurements could be made with light focused at points along a grid covering the entire photocathodes. A portion of the incident light was split from the incident beam and measured and recorded by a fast photodiode. Electron transit times were measured by computing the time delay between the recorded photodiode signal and photomultiplier signal using software constant-fraction discrimination. The Hamamatsu tube exhibited a uniform timing response that varied by no more than $1.7 \mathrm{~ns}$. The Adit tube was much less uniform, with transit times that varied by as much as $57 \mathrm{~ns}$. The Adit response also exhibited a spatially varying double-peak structure in its response. The technique described in this paper could be usefully employed by photomultiplier tube manufacturers to characterize the performance of their products.

- Keywords: Photomultiplier tubes, Detector calibration methods

\section{Introduction}

Photomultiplier tubes (PMT) are often used with scintillation detectors in applications where timing is important, such as in coincidence or time-of-flight

ts

Preprint submitted to Nuclear Instruments and Methods in Physics Research Section AApril 18, 2016 
experiments, or in positron emission tomography (PET) [1]. In these applications, the electron transit time in the PMT usually contributes the largest temporal variation to the overall system. Photons arriving at different locations on the photocathode produce photoelectrons with varying trajectories through the tube, which results in timing variations in the output signal. This timing variation becomes more pronounced as the size of the PMTs increases. In experiments where the light signals illuminate the photocathode uniformly, the timing and shape of the electronic output pulse tends to be fairly uniform between events. However, localized illumination of the photocathode results in variations in shape and timing of the response pulses with the location of the input light. In experiments with localized illumination, such as in low-photon counting or when the PMT is coupled to optically isolated detector segments, it is important to understand the relationship between PMT timing and the location of the incident light on the photocathode.

Our interest in the spatial variation of the time response came from doing energy-dependent efficiency calibrations of neutron detectors using time-of-flight techniques. The detectors involved were capture-gated detectors that were constructed with optically isolated sections of scintillator surrounded by cadmium foil. We originally used Adit B133D01S PMTs, but found the time-of-flight data to be minimally useful because of the timing spread of the detectors.

Researchers at UCLA investigated the timing characteristics of a 3 -inch Hamamatsu R11410-10 tube used in a vacuum cryostat for Dark Matter detection experiments. They found that the transit time was highly position dependent, with deviations of about 5 ns near the edges of the PMT [2]. They also found symmetry along the axes corresponding to the location and design of the first dynode. In an unpublished study, Xu Wang and Ye Tian at Shandong University investigated several 1-inch and 2-inch PMTs for applications in cosmic ray astronomy. Their initial results confirm those of the UCLA study. In addition, they found that locations with a slower relative transit time, such as the edges, also have greater transit time spread among the samples taken at that location. Xia et al. have studied the spatial uniformity of the sensi- 


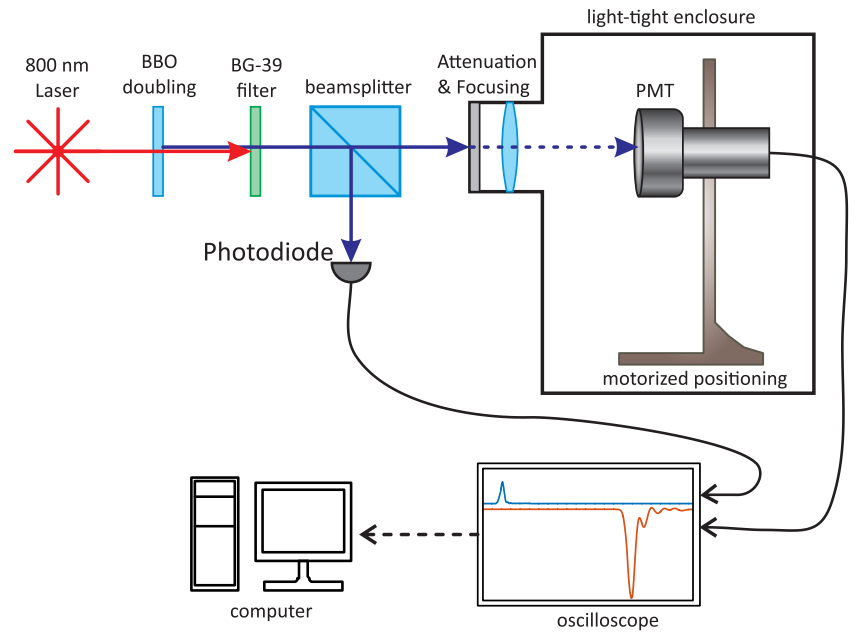

Figure 1: Schematic of the measurement setup. An $800 \mathrm{~nm}$ laser pulse is frequency-doubled in a $\mathrm{BBO}$ crystal to produce $400 \mathrm{~nm}$ light pulses. The $800 \mathrm{~nm}$ laser pulse is removed using a BG-39 spectral filter, and the $400 \mathrm{~nm}$ pulses are attenuated and focused onto a PMT. A photodiode triggers the oscilloscope, and a computer records each PMT output pulse. tivity 1-inch and 2-inch PMTs, and separately looked at their timing response (although they did not study the spatially resolved timing response) [3].

These prior results were each obtained for tubes 2-inches or less. In this paper, we present results for the timing behavior of 5-inch diameter PMTs. These larger tubes have the potential for larger timing variations due to the larger geometry, and timing studies have not yet been reported.

\section{Experimental Procedures}

A schematic of our experiment is shown in Fig. 1. A Ti-Sapphire laser produces 100 ps pulses at $800 \mathrm{~nm}$, which are frequency doubled in a Beta Barium Borate (BBO) crystal to produce $400 \mathrm{~nm}$ pulses. These $400 \mathrm{~nm}$ pulses are a good match for the PMTs used in this measurement, which have a peak sensitivity wavelength about 420-460 nm. The laser pulses then pass through a Schott BG39 filter to remove the $800 \mathrm{~nm}$ light, and a beamsplitter diverts a portion of the beam to a fast photodiode (ThorLabs DET210) used to trigger the oscilloscope. 


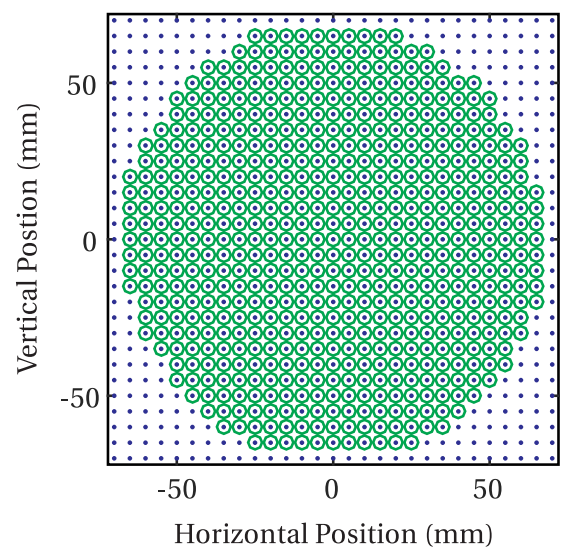

Figure 2: The dots represent the PMT positions at which data were recorded. The circled dots are locations where PMT signal were detected.

The remainder of the light is attenuated with neutral density filters and focused using a lens onto the surface of the PMT at normal incidence, with a spot less than $2 \mathrm{~mm}$ in diameter. An oscilloscope digitizes both the PMT output pulse and the trigger pulse from the fast photodiode, and both traces are then saved to a computer for later data analysis.

A pair of computer-controlled linear stages translate the PMT in the plane parallel to the photocathode to vary the spatial location where the optical pulse strikes the PMT. The PMT is oriented such that features of the interior geometry are aligned to the axes of the stages. Data are taken on a rectangular grid over the entire face of each photocathode with $5 \mathrm{~mm}$ spacing in both dimensions. The positioning uncertainty of the stage is about $10 \mu \mathrm{m}$. We verified the location of the PMT by spatially scanning over our square grid. When the laser pulse position was outside the active area of the PMT, no PMT pulse was produced. This allowed us to verify the location of the tube within the scanned grid. The dots in Fig. 2 show the locations where data were taken, and the circled dots are positions where signal was observed.

We used this system to measure the timing characteristics of two 5-inch PMTs: an Adit B133D01S and a Hamamatsu R1250. The Hamamatsu tube is designed to optimize timing response, while the Adit tube is engineered to op- 
timize detection efficiency. Both tubes are head-on type PMTs that use bialkali photocathodes with a minimum useful diameter of $120 \mathrm{~mm}$. The Hamamatsu employs a 14-stage linear focused dynode structure with a maximum operating voltage of $2000 \mathrm{~V}$. The Adit has a 10-stage box-and-grid dynode structure with a maximum operating voltage of $1500 \mathrm{~V}$. In these measurements, the PMT voltages were set near their maximum voltages. To prevent saturation, we attenuate the incoming laser pulses by inserting sufficient neutral density filters in the beam to just eliminate the PMT signal, and then remove a filter with optical density one to restore the signal. The Hamamatsu PMT has a larger gain, so the Hamamatsu PMT requires more attenuation than the Adit.

\section{Analysis}

The primary goal in this study is to characterize the relative timing between the fast photodiode trigger pulse and the photomultiplier tube response. The intensity of the Ti-Saphire laser pulses varies shot-to-shot by $20 \%$, and the efficiency of the frequency doubling process is quadratic with intensity. This results in fluctuations in peak height for both the fast photodiode and the PMT response. These fluctuations can affect the relative timing between the two signals when a simple voltage threshold method is used to identify pulse arrival times for the two pulses. To remove this effect, we employed a software constant fraction discrimination (CFD) algorithm to the recorded trigger pulse to adjust the relative timing. This process adds an inverted, attenuated pulse to the original trigger pulse and adjusts the time axis so that the zero-crossing of the summed pulse gives the arrival time of the pulse, as illustrated in Fig. 3. The CFD algorithm reduces jitter that arises from variation in pulse size.

The formula for the summed pulse in the CFD algorithm can be written as

$$
S(t)=P\left(t-t_{d}\right)-\alpha P(t)
$$

where $S(t)$ is the summed pulse, $P(t)$ is the original pulse, $\alpha$ is the attenuation factor, and $t_{d}$ is the delay time. We found that for single-peak pulse shapes in 


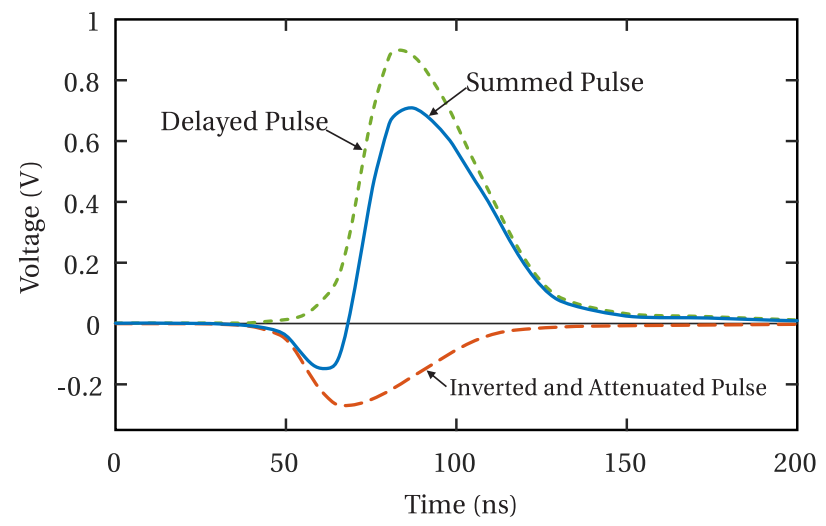

Figure 3: A schematic representation of the process used to determine timing with a CFD algorithm.

our experiment there was very little sensitivity to the choice of $\alpha$ or $t_{d}$ within the reasonable choices for these parameters. However, the Adit tube exhibited a distinctive double-peak structure in its response. For this type of double-peak structure, the $\alpha$ parameter can be varied to distinguish the arrival of the two peaks.

Typical response curves for our setup shown in Fig. 4 for the Hamamatsu and Fig. 5 for the Adit. Note the small peak preceding the main response in the Adit response curve. This double-peak behavior occurred when testing certain regions of the PMT and not in others. This suggests a bifurcation of the transit paths of electrons through the PMT amplification chain, but it is unclear whether or not single photons would produce a double-peaked pulse. For double-peaked pulses, small values of $\alpha$ pick the timing from the first peak and larger values from the second peak. We analyze the data using both $\alpha=0.1$ and $\alpha=0.9$ for the Adit tube to separately study the timing of the two types of electron trajectories. With the Hamamatsu tube, we only display the analysis done with $\alpha=0.1$. 


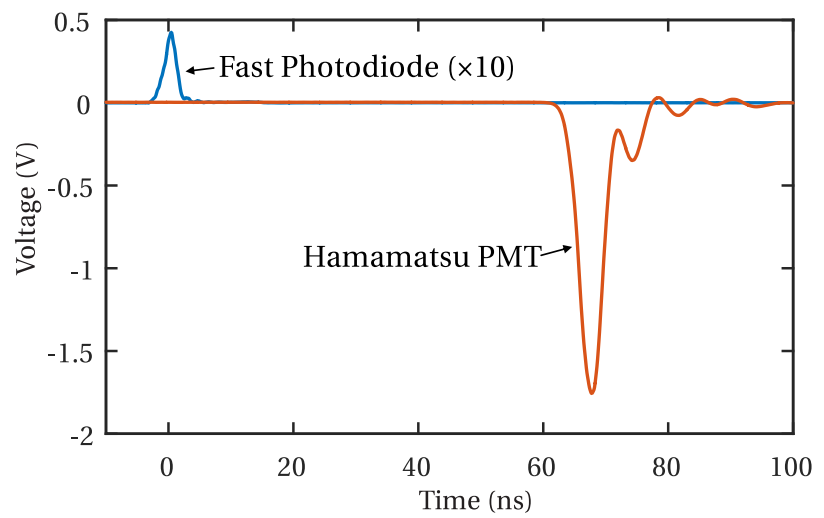

Figure 4: Typical response for the Hamamatsu PMT. The triggering fast photodiode is also shown for comparison. The peak voltage of the fast photodiode signal has a significantly smaller amplitude than the PMT, so for plotting purposes we have multiplied the fast photodiode voltage by ten to aid visual comparison of the two peaks.

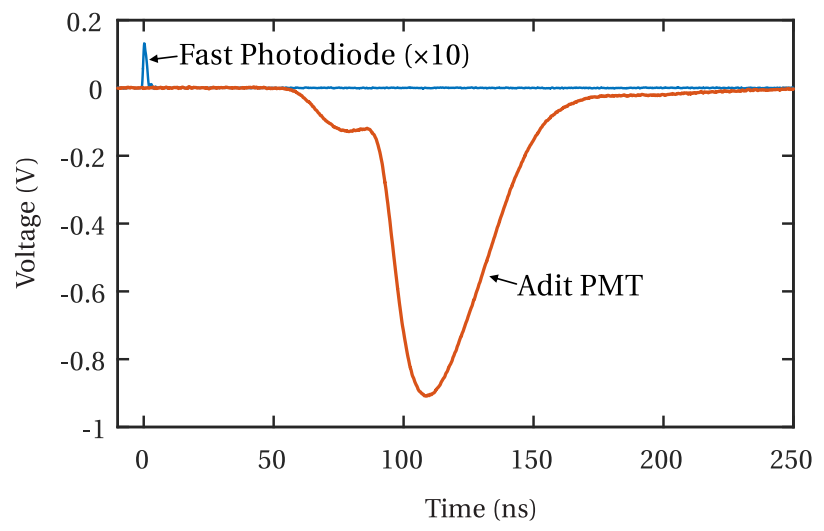

Figure 5: Typical pulse for the Adit PMT. The triggering fast photodiode is also shown for comparison. The peak voltage of the fast photodiode signal has a significantly smaller amplitude than the PMT, so for plotting purposes we have multiplied the fast photodiode voltage by ten to aid visual comparison of the two peaks. 


\section{Results}

Although the light incident on the photocathode is localized, the photoelectrons ejected from the photocathode still exit with a range of angles and energies. These variations result in shot-to-shot fluctuations in timing at a given position. To average out this effect, we measure ten pulses at each location on the grid Then we calculate the difference in time between the PMT pulse and the fast photodiode pulse, $\Delta t$, for each laser pulse and average the values of $\Delta t$ for each spatial point.

The calculated values of the time delay $\Delta t$ between the photodiode trigger and the PMT response include an arbitrary constant that depends on cable and electronic delays. To remove this constant, we subtract the average value of all valid measurements, $\langle\Delta t\rangle$ from all the individual measurements of $\Delta t$ in our results. The average values $\langle\Delta t\rangle$ are of some interest as a comparison between the different measurements, however. For the Adit tube with $\alpha=0.1$, the CFD algorithm gives the arrival time of the first peak. The average arrival time $\Delta t$ of this first peak is $83.5 \mathrm{~ns}$ with a standard deviation of $11.7 \mathrm{~ns}$. When we use $\alpha=0.9$, the CFD algorithm gives the arrival time of the second peak. The average arrival time of the second peak is $111.7 \mathrm{~ns}$ with a standard deviation of 14.5 ns. Biasing the data toward the later peak increases the measured value of the transit time, as expected, but it also increases the transit time spread.

To spatially resolve the transit times, the procedure described above is applied to the data at each location. Figure 6(a) shows a map of the delay time as a function of position for the Adit tube with $\alpha=0.1$, which selects for the early peak. Figure 6(b) plots a histogram of transit times at all positions. The largest peak in the histogram occurs at about $8 \mathrm{~ns}$, which correspond to the large outer portion of the PMT as seen in Fig. 6(a). The inner portion of the PMT has much shorter delay times, corresponding to the lower portion of the histogram. Note the annular region about $15 \mathrm{~mm}$ from the center where there is an early double peak leading to shorter transit times.

When we switch to $\alpha=0.9$ for the Adit tube, we consistently take our timing 

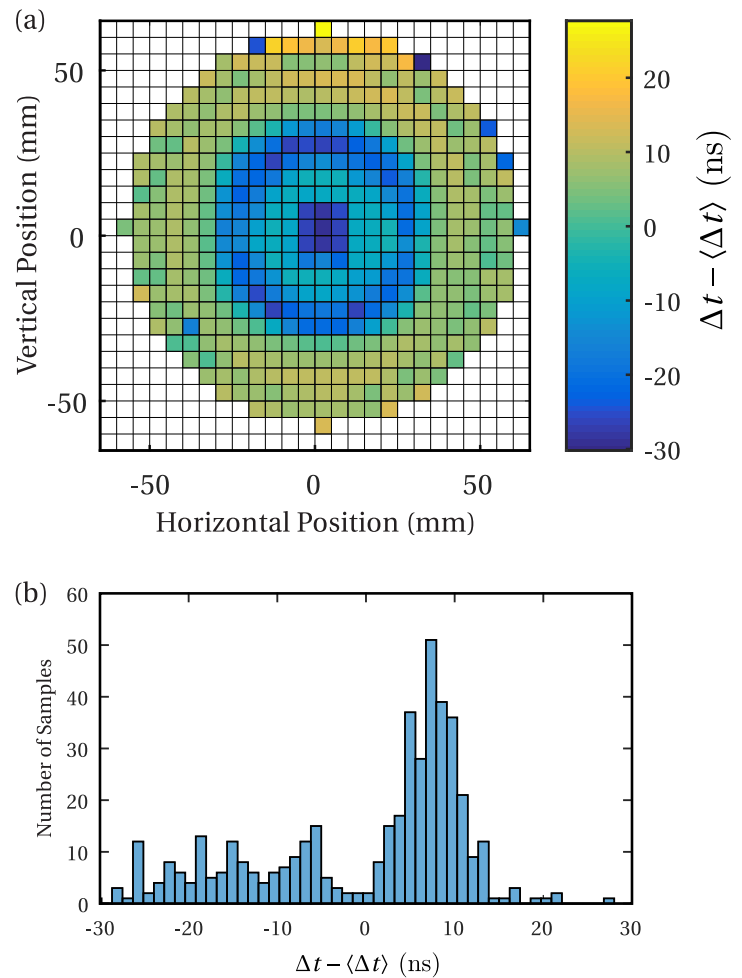

Figure 6: (a) Difference of transit time and mean transit time as a function of the position of optical stimulation for the Adit tube with $\alpha=0.1$. (b) Histogram of the transit time minus the mean transit time for the Adit tube with $\alpha=0.1$. 

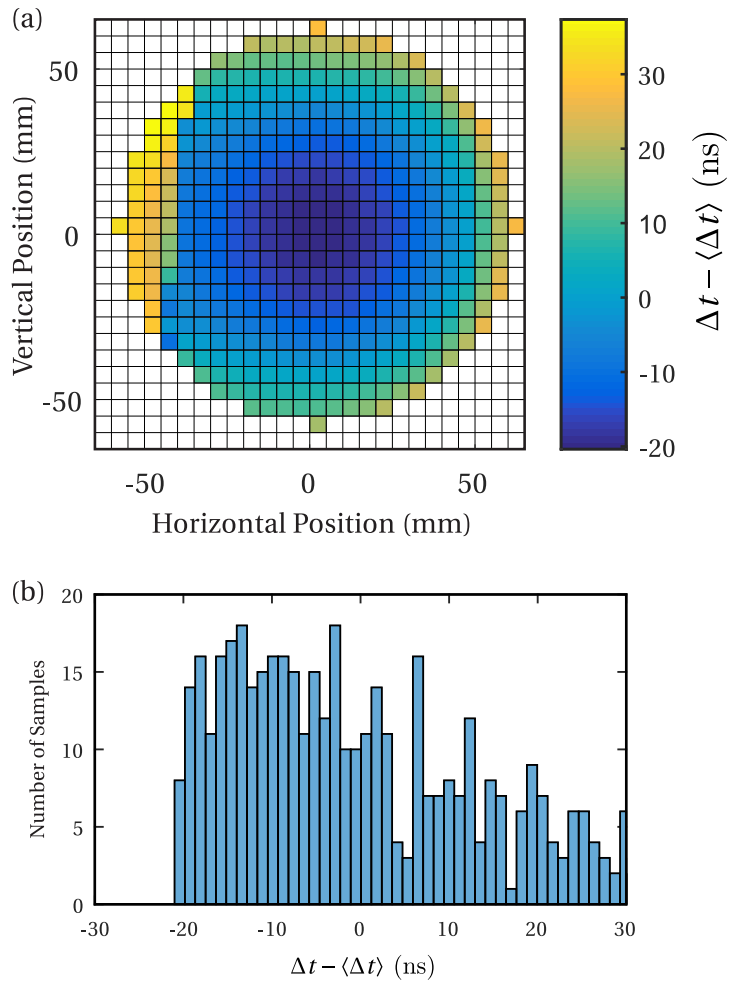

Figure 7: (a) Difference of transit time and mean transit time as a function of the position of optical stimulation for the Adit tube with $\alpha=0.9$. (b) Histogram of the transit time minus the mean transit time for the Adit tube with $\alpha=0.9$.

information from the later peak. Figure 7(a) plots a map of the delay time as a function of position for $\alpha=0.9$, and Fig. 7(b) plots a histogram of transit times. The histogram shows a wide distribution in transit times. If plotted as a surface, the data in Fig. 7(a) resembles a paraboloid with a large variation in transit times from the center to the edge. The shortest transit times are near the middle, with the delay time increasing by over $50 \mathrm{~ns}$ as the laser position is shifted to the edge of the PMT.

We expected that the timing characteristics of the Hamamatsu tube would be much better than the Adit tube, and this is borne out in the data. A spatial map of the delay times for the Hamamatsu tube is plotted in Fig. 8(a) for $\alpha=0.1$, and a histogram of the delay times is plotted in Fig. 8(b). The spatial 

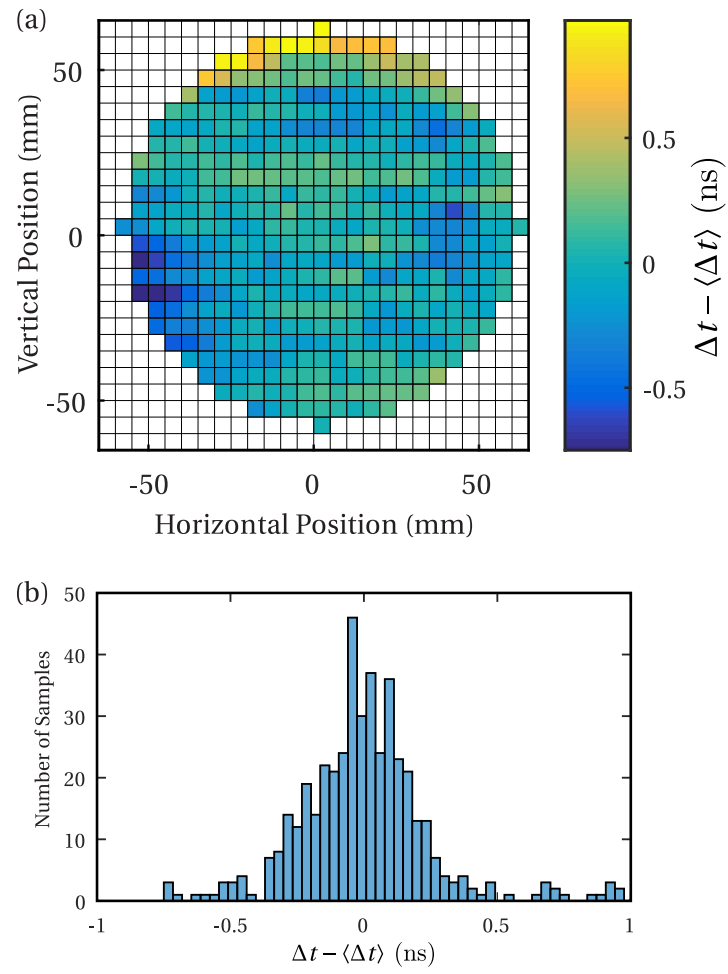

Figure 8: (a) Difference of transit time and mean transit time as a function of the position of optical stimulation for the Hamamatsu tube. Note that the color scale for this plot represents a very small range of transit times (only about $1 \mathrm{~ns}$ of variation), so the structure visible in the plot is mostly noise. In contrast, the variation for the Adit tube was several tens of nanoseconds, as seen in the level scales for Figs. 6 and 7. (b) Histogram of the transit time minus the mean transit time for the Hamamatsu tube.

map shows that the timing response is very spatially uniform with a few noiselevel artifacts. There is a slightly longer delay time just at the edge of the PMT, but this effect is very minor compared to the Adit tube. The average delay $\langle\Delta t\rangle$ was $63.6 \mathrm{~ns}$. The standard deviation for the distribution shown in the histogram is $0.2 \mathrm{~ns}$, and the distribution has a FWHM of $0.4 \mathrm{~ns}$. Thus, the Hamamatsu tube has a shorter transit time than the Adit, and minimal spread in transit times. 


\section{Conclusions}

We have investigated the transit time variations for electrons produced by photons originating at different locations on the photocathodes of two commercial 5-inch PMTs: the Hamamatsu R1250 and the Adit B133D01. The Adit tube exhibited significant spatial variations in transit time, while the Hamamatsu was relatively uniform. The Hamamatsu tube, which is engineered for excellent timing properties, performed much better than the Adit tube. The distributions of transit times for the Hamamatsu tube varied with a FWHM of only 0.4 ns. Within the minimum useful diameter, transit times in the Adit tube varied by as much as $57 \mathrm{~ns}$ from one location to another. Furthermore the double peaking of the Adit tube requires care when analyzing timing pulses, especially when localized optical signals are detected on various portions of the detector. It would be helpful for users if manufacturers provided this type of timing analysis.

\section{Acknowledgements}

Parts of this work were funded by NNSA Grant DE-FG52-10NA29655 and DHS Award 2010-DN-077-ARI039-02.

[1] Tomasz Szczesniak et al., "Fast Photomultipliers for TOF PET," IEEE Trans.Nucl.Sci. 56 (1), 173-181 (2009).

[2] K. Lung et al., "Characterization of the Hamamatsu R11410-10 3-in. photomultiplier tube for liquid xenon dark matter direct detection experiments," Nucl. Instr. Meth. Phys. Res. A 696, 32-39 (2012)

[3] J. Xia et al., "A performance evaluation system for photomultiplier tubes," J. Instrum. 10, P03023 (2015).

[4] Xu Wang and Ye Tian, "A study of head-on type photomultiplier tubes," Shandong University Unpublished Apr 21 2014, URL accessed July 19, 2014 no longer available. 